\title{
Hydrobiologische Referat review of Woltereck (1909)
}

\author{
LUdWIG KeILHACK* \\ Zoologische Institut der Universitaet Berlin \\ maximilian@alumni.reed.edu
}

April 22, 2020

\begin{abstract}
A contemporaneous review of Woltereck's paper published closely thereafter critically evaluating the work in comparison to Johannsen. Originally published 1909 in Internationale Revue der gesamten Hydrobiologie und Hydrographie Bd.11. H. 4 u. 5. pp. 770-778. Note that Woltereck was a founder of and frequent contributor to this same journal; we may therefore consider it to be relatively friendly to him.
\end{abstract}

The investigations with beans and barley races by the Danish botanist Johannsen (among others) have demonstrated that an apparently uniform stand (a "population") can in actuality be a mixture of different types, each of these rather more uniform and stable. $\mathrm{He}$ showed this through culture experiments "in pure lines", which is to say in successions of generation in which plants are derived solely from self-fertilization or parthenogenesis. Johannsen found the following: 1 . The specific structure that these elementary types ("biotypes") inherit in their germplasm ("genotypical basis" or "genotype") are fixed quantities, that over the course of generations do not vary in the least. Change of these structures can only be achieved through spontaneous mutations. 2 . The direction of these mutations has no relation to the environmental circumstances. For Johannsen, there is no continuous evolution and no definitive influence of the environment.

Together with the discoveries of De Vries regarding mutations and Mendel's crosses, these propositions are apt to seriously threaten the notion of continuous variation under the decisive influence of the environment. The author [MOP: meaning Woltereck] seeks by means of analytical experiments to test Johannsen's propo-

*translation by Maximilian Oliver Press 2020 sitions, and if necessary to limit or improve them.

For this purpose the Daphnia (specifically the genus Daphnid ${ }^{1}$ provide an excellent material. The numerous landraces are heritably different, mostly uniform populations that by many generations of continuous parthenogenetic reproduction can be cultured in pure lines. The various biotypes provide a variety of quantitative characters (for example length of the shell spine, head-height, forehead angle, spine angle, degree of sexuality) that are amenable to exact measurement. There is no difficulty in obtaining the elementary types: all the parthenogenetically derived descendants of a single $q$ represent a single uniform type.

The author here makes an interesting observation: most of the investigated landraces appear to be uniform populations; but in spite of this an apparently uniform population of Daphnia longispina-galeata proved to be composed of two biotypes. This behavior is probably similar across many cladoceran populations. In cladocerans all forms exist between obligatory amphigony at least once a year (for generating dauer eggs) and complete suppression of sexuality, so it is excellent that we can study the

\footnotetext{
${ }^{1}$ I call the genus Daphne, but here I follow the author's nomenclature.
} 
influence of amphigony on the uniformity of populations.

The author tests Johannsen's propositions using the parthenogenetic descendants of single individuals. He fully directs the investigation through for the two characters of helm-height and sexuality. The plan of work is as follows:

1. Determination of the entire breadth of variation of a character under all practical environmental conditions.

2. Determination of the environmental quality that brings forth the relevant modification of each character.

3. Determination of the heritable differences of the collected modifications of a character that different biotypes show under the same environmental conditions.

4. Finally, to test whether and how far the persistent influence of the environment can heritably alter the characters of a biotype.

The behavior of two biotypes under crossing is seen to be a further, technically much more difficult area of investigation. The author found by and large intermediate behavior, but has not come to any final conclusion. The same was true regarding the question of whether persistent selection of extreme variants can change a biotype. In most cases, like Johannsen, this question was answered in the negative ${ }^{2}$

\section{HeLM-HEIGHT}

This character shows itself to be dependent on the external factor of nutrition and the inner factor of generation number.

Temperature, which had been given by Ostwald as the decisive factor, was shown to play only a secondary role in that it influenced nutrition. Ostwald's conception is decisively refuted by the author's work. The quince mucilage

\footnotetext{
${ }^{2}$ MOP: my emphasis.
}

experiment $\mathrm{s}^{3}$ of the author also addressed Ostwald's erroneous perception that the inner rubbing of the water created a direct stimulus for the morphological modifications. To my knowledge, this is not the case.

Helm-height is directly proportional to assimilation, and thus is the exact control approachable: in cultures for which temperature, salt content, gas content, volume, and light are carefully controlled, there remains only the external factor of food supply, which the author can readily dose. The inner factor of "generation count" is a cyclical variable component of the otherwise constant heritable structure of helm development, the "helm-potential".

The author gives a complete description of the development of the helm-building region and the physiological assimilation processes, that act as a regulating stimulus for helm development of the embryo and helm growth in the pregnant animal.

In order to learn more about the peculiarities of helm development of a biotype, the author ascertained the middle ${ }^{4}$ helm-height in different generations $a b$ ephippio and for different food quantities, in the following nine cases:

Helm height in the beginning generations with poor, middling, and rich nutrition.

Helm height in the middle generations with poor, middling, and rich nutrition.

Helm height in the late generations with poor, middling, and rich nutrition.

Some biotypes that react differently while receiving similar amounts of food but different temperatures must in each of these nine case also be investigated at low, middle, and high temperatures in order to get an approximate idea of their specific reactions for a character. A general representation of the entire set of numerical data can be found in the "phenotype curves' 15 , which display the assimilation factor

\footnotetext{
${ }^{3}$ MOP: what a wonderful word: Quittenschleimversuche!

${ }^{4} \mathrm{MOP}$ : arithmetic mean.

"Phenotype" is what Johannsen names the statistically distinguishable type, for which it cannot yet be said whether it is an expression of a biological uniformity or not. Woltereck understands this as the mean value of a character for a defined environment; the term "phenotypic difference" he uses in the same sense as Johannsen;
} 


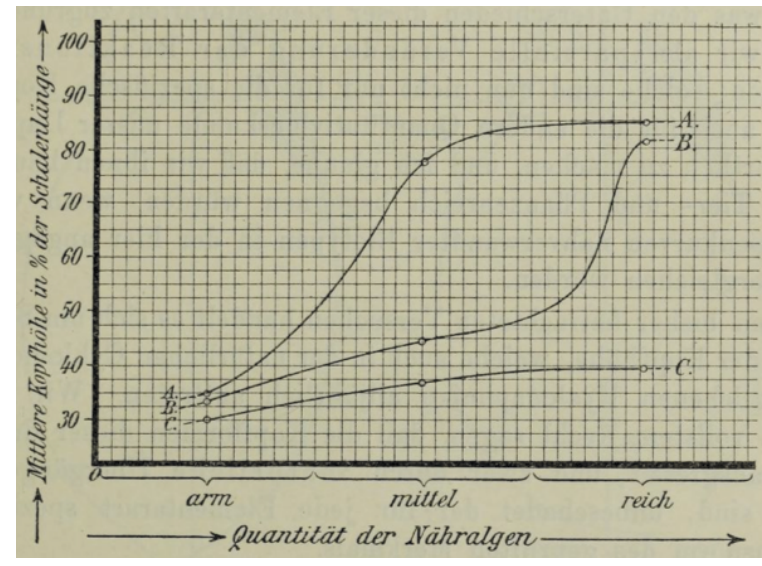

Figure 1: Phenotype curves of relative head-height for three biotypes (local varieties; $A, B, C$ ) of Hyalodaphnia cucullata.

or the generation number on the abscissa and the height of the helmet as a percentage of the mantle length.

The totality of these curves yield a picture of the specific "reaction norm" of the repsective biotype; (Johannsen speaks instead of the "genotype" or the "genotypical basis"). Therefore, the following two propositions hold for the Daphnia types: first, the entire reaction norm with all its innumerable specific reactions is inherited in the helm structure; and second, the biotypes of Daphnia arise and have arisen through heritable alterations in the reaction norm of their helm height and their other characters. The question now stands whether the controversial propositions of Johannsen also hold for this reaction norm. The author next refers to a mutation that arose in his cultures of Daphnia longispina from the Lunz Untersee, whose phenotype curve has at all points a similar distance from that of the principal form. It appears that here we see a typical mutation (spontaneous arisen without influence of the environment, and heritable), whose incidence is thus noticeable in the Daphnia.

In contrast to this example we may show the phenotype curves of three closely-related biotypes of Hyalodaphnia cucullata with the following behavior: for poor nutrition, negligible

therefore he does desire an alteration of this term. helms for all three, A the largest and $C$ the smallest; for middling nutrition, slightly increased helms for two (B and C) and much higher helm for the third (A); for rich food, yet higher helm for $\mathrm{A}$, strongly increased helm for $B$ that almost reaches $A$, and for $C$ barely any alteration of the helm height. The curves show, when observed pairwise, all transitions from stark divergence to near absence of any difference. For these there also exist partial differences of the reaction norm, which surely indicate the occurrence of partial alterations of the reaction norm (the genotype), whereas for Johannsen only saltational and therefore total modifications of the genotype play any role. Additionally, Woltereck's phenotype curves clearly announce "environmental dependence" of these quantitative racial differences, again in contrast to Johannsen.

For two elementary types from the Lunz Untersee and Lunz Obersee, the author could not compensate for the difference in conditions of life through substitution, except through application of overwhelming alteration of life history: the fat form from the Obersee was similar to the Untersee form in starved cultures, and the hungry form of the Untersee could be altered by overwhelmingly rich food into the Obersee Daphnia. Additionally, all possible transitions between these forms appear. These transitions indicate 1 . that the differences in character between the two forms are not arisen discontinuously through mutation, and 2. that this emergence of difference is brought forth by the environment. Therefore, continuous variation with pronounced environmental action. Finally, we must yet recall that for some elementary types, the reaction norm is different for different generations. It is apparent that the first generations after the dauer egg develop a smaller helm than the middle generations with similar feeding. Here there is a heritably fixed diminution or confounding of helm development for the first (spring) generations, which are acclimated to poor nutrition. Here also a distinct action of the environment is apparent.

We can at this point distinguish three stages of the divergence of biotypes: 1. partial al- 
teration of the reaction norm, given natural transitions (example: the biotypes A and B of Hyalodaphnia, see above); 2. natural transitions are missing; however, through transplant to unnatural environmental conditions we can obtain artificial transitional forms (example: $D$. longispina from the Lunz Untersee/Obersee); 3. the reaction is different throughout, and transitions can no longer be brought forth artificially (example: the biotypes $\mathrm{A}$ and $\mathrm{C}$ of Hyalodaphnia, see above).

\section{SexuAlity}

In Daphnia, we define sexuality as the ability to produce dauer eggs and males, rather than parthenogenetically conceived females. We may evaluate this quantitatively by using the percentage of males in the generation or dauer eggs in the clutch as a criterion. The character of sexuality is dependent upon the same factors as those exerting influence on helm-height; these sexual differences of the biotypes are continuous and environmentally determined. The proof for this was achieved in the same ways as for helm-height: through testing of natural and artificial transitions and through comparison of different generations.

\section{i. Natural transitions:}

The middle generations of three biotypes showed the following grades of sexuality: all three responded to undernourishment with full $(100 \%)$ sexuality, to overnourishment with overwhelming parthenogenesis, and under normal (middling) nourishment the first form had 50\% sexuality, the next had $75 \%$, and the third had 100\% sexuality. Therefore: partially different reaction norms with natural transitions, exactly as with helm-height.

\section{ii. Artificial environment-determined transitions:}

The author succeeded in converting to sustained parthenogenesis a Daphnia pulex (obtusa) from the Lunz alpine ponds (elevation
$1600-1800 \mathrm{~m}$ ), that follows a short, sharply defined cycle in the wild ${ }^{6}$ He has thereby accomplished a transition between two extremes: the short cycle of the high mountain Cladocerans and the acyclic reproduction of many lake dwellers. Pure parthenogenesis is in both cases dependent on similar environmental factors (sustained rich feeding).

\section{iii. Reaction differences of genera- tions, in agreement with the natural environmental relations:}

Among the many diverse generational progressions of sexuality that appear among the Cladocerans, we can distinguish four through transitions:

First category: Sexuality increases rapidly in the course of the smaller (first, second, or third) generations from $0 \%$ to $100 \%$ and then becomes a) obligatory (completely dominant under all circumstances), or b) remains facultative and partial, i.e. they can through rich feeding tilt the balance towards parthenogenesis (in different circumstances).

Second category: Sexuality increases gradually in the course of higher generations (10 or more) from $0 \%$ to $100 \%$; a) and b) as with category 1 .

Third category: Sexuality increases nearly as fast, without becoming general; the descendants of those 9 of derived from remaining or reverting parthenogenesis remain completely asexual; then gradually sexuality reasserts itself, and becomes a) after 6-12 generations absolutely obligatory or $b$ ) stays facultative and partial.

Fourth category: All generations are completely and obligatorily parthenogenetic, which

\footnotetext{
${ }^{6}$ Here and throughout my opinion is contradicted, that the pond-dwelling Cladocerans with short cycles (see $p$. 239 of this volume) are maintained to this by good feeding, my three propositions (see there) are no longer to be supported.
} 
is to say that no sexuality arises even under conditions of low assimilation.

The author portrays the behavior of three forms in his cultures: 1. a Hyalodaphnia $\mathrm{cu}$ cullata race belonging to category 2 a showed obligatory parthenogenesis in the first generation after ephippium, and in the following an increasing tendency towards sexuality, that finally became obligatory in all grades of environment (see Fig. 2). 2. Under the author's guidance V. Scharffenberg experimented with Daphnia magna (category 1), and found a quickly increasing tendency towards sexuality not only changed from one generation, but also the same individuals from a single clutch could change. He further showed that of hatched directly from $D$. pulex dauer eggs after a series of parthenogenetic broods can already develop dauer eggs. The production of $\sigma^{7} o^{7}$ was unaffected by different grades of nutrition in his (and O. Kuttner's7) observations. 3. A dicyclic D. galeata (category 3 ) already shows a strong sexual tendency in the second generation after ephippium (60\% with good nutrition), and is followed by more asexual generations and then a sudden increase of sexuality $(80-90 \%$ by good nutrition). The table of categories and the examples show sufficiently that the manifold forms of generational progression and their numerous transitions cannot be considered as derived spontaneously.

The dependence of the various forms of reproductive methods on natural environmental relations appears clearly in most cases.

The polycyclic forms (category 1) inhabit small bodies of water, in which after only short times shortages of food and oxygen appear, which along with enrichment of carbon dioxide and azotically rich excretions brings an end to assimilation. "This cycle: dauer eggs - 1 to 3 parthenogenetic generations - dauer eggs are the substrate (gonadal primordia and germ plasm) in which this reaction process plays itself out, forced firmly in this way in the course of very many generational cycles, such that today in many cases even without

\footnotetext{
${ }^{7}$ Compare the article of O. Kuttner (Freiburg) in this volume, that of $U$. von Scharffenberg in the next.
}

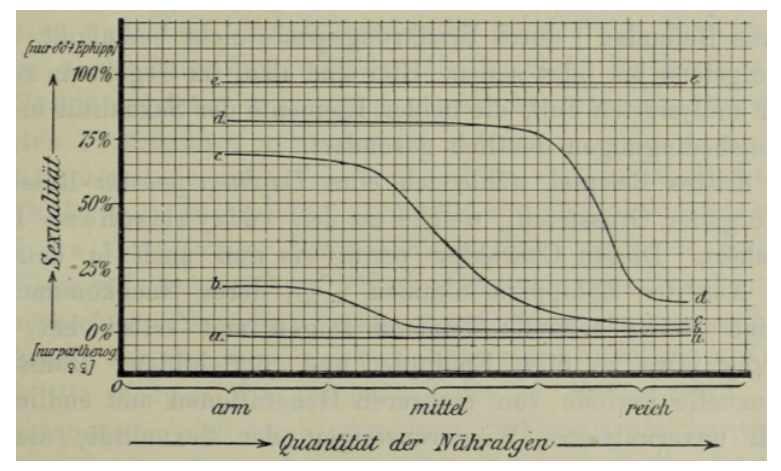

Figure 2: Phenotype curves of sexuality for different generations of a single Hyalodaphnia race. $a$, first generation after ephippium; $b$-d middle generations; e, last generation.

the habituated increase and decrease of the assimilation agents, if also mostly in moderated forms (facultative or partial sexuality also in the later generations, apart from the total sexuality, which is then maintained openly). 8 Thus, here we find a heritably fixed character whose appearance is evidently dependent on the environment.

Besides the precisely-named assimilation, another second cause may work towards the fixation of the generational cycles: natural selection, which Weismann correctly preaches. The eminently suitable alternation between rapid parthenogenetic reproduction and the development of drought- and frost-resistant dauer eggs leads the author back to their influence 9 "The appearance of parthenogenesis-so useful for rapid proliferation-from the originally sexual reproduction is likewise attributable to selection, as the fine structural design of the ephippia with their exquisite functionality.' 10

In this reviewer's opinion, these two causes do not work in parallel, but rather selection the singular principal cause in engendering this suitability. The ability to answer poor assimilation stimuli with the development of resistant

\footnotetext{
${ }^{8}$ MOP: quote from Woltereck's section "First category of generational progressions".

${ }^{9} \mathrm{MOP}$ : throughout this article but here especially Keilhack is copying phrases directly from Woltereck in a piecemeal fashion, even when he isn't directly quoting.

${ }^{10} \mathrm{MOP}$ : quoted from the same section of Woltereck
} 
dauer germs, and rich assimilation with rapid parthenogenetic reproduction, is acquired under the influence of selection. The assimilation is no cause of the suitability of the reproductive cycles, but rather the means by which selection addresses these circumstances appropriately.

For the second category, the monocyclic cladocerans with dauer egg development in autumn, the initiation of dauer egg development in cultures represents an acclimation to the return of a highly predictable autumnal decline in assimilation. The environmental dependence is clearly with regard to the end of the cycle. Less simple is the explanation for the behavior of the first (spring) generation; they are completely asexual, in spite of the quite poor rate of assimilation of their environment. The author posits one possible explanation, though it does not seem adequate to this reviewer. It concerns the very convenient weakening of the accommodated reaction, which explanation we would anyways not obtain without selection.

The third category creates some difficulties: the dicyclic mode of reproduction of cladoceran colonies in large bodies of water cannot be explained by reference to the obvious characteristics of the natural environment. There are two possibilities for explanation: the generational progressions of these colonies are a reminiscence of an originally smaller water habitat or an originally more arctic climate. (The author doubts the first, the referent doubts the second notion, at least for the typical lake inhabitants with dicyclic reproduction ${ }^{11}$. It seems simpler to me to think that a modification of the generational progression will go hand in hand with the slow modification of the habitat, than to make a change of the habitat and thereby the conditions of life responsible for the alteration of the generational cycle. The dicyclic mode of reproduction may be demonstrated otherwise in a very typical development for two cladocerans, Polyphemus pediculus and Bosmina longirostris, which both inhabit larger waters by preference. If their

\footnotetext{
${ }^{11}$ For the behavior of a $D$. longispina from the Schwielowsee (Havel), I myself once gave the first-named explanation.
}

generational cycles were a reminiscence of an originally smaller body of water, then we must still explain their present near absence in these same.

I will take the opportunity to note that for the selfsame Bosmina the first (June) sexual period is more distinctly marked than that in October (both are only partial); this type may yield important information concerning the action of the assimilation stimulus and its limits through culture experiments.

For the fourth category the action of the environment is completely obvious: the inhabitants of the planktonic zone of our great waters stand richly provided with food the entire year, and have sometimes completely given up the sexual period and now fail to react to artificial nutritional stimulus with sexuality: their reproductive mode is stiffened to pure parthenogenesis. Here, beside the pelagic types of the genus Daphnia and Bosmina, there also belongs a colony of D. pulez from the warm lake of the Astroni crater in the Phlegraic fields, which at least in the cultures of the author have demonstrated parthenogenesis for many years.

Therefore, the author opines that the character "sexuality" shows an interpretable influence of the environment. The second and third categories give this interpretation considerable difficulties, and for the present this reviewer does not share his optimism.

\section{The Emergence of Regressive VARIANTS}

The author studies the emergence of regressive variants in two characters: the nebenauge ${ }^{12}$ of Hyalodaphnia and also its crown toothlet. At the same time, he presents experiments towards their artificial production and fixation.

The nebenauge, whose presence and absence the author believed himself to have proved a mutationally derived character, shows with closer examination all transitional forms from the complete structure all the way to complete absence. Also, through his experiments he

\footnotetext{
${ }^{12}$ MOP: the German term appears to be the accepted one in English usage.
} 
could ascertain the previously conjectured behavior of single generations concerning the development of the nebenauge. Specifically, in the first generation from dauer eggs the nebenauge showed itself more frequently than in the later generations. The transitions showed a degree of heritability: while in many pure lines the nebenauge appeared randomly and disappeared again, the author could in one case measure a $90 \%$ heritability of the character. The author could not demonstrate any influence of the environment on the nebenauge.

The crown toothlet also showed all transitions 1. in grade of development, 2. in frequency of appearance, 3. in its heritability, and 4 . in the different strong tendencies of different generations. Otherwise however it allowed the author a more obvious (if also in its causality less comprehensible) influence of the environment: if ofo with ripe eggs in the ovary are given a sudden temperature shock by transfer from $25^{\circ} \mathrm{C}$ to $12^{\circ} \mathrm{C}$, then their offspring will show the crown toothlet.

Both regressive characters show continuous modes of appearance, just as for helm height and sexuality. The one (crown toothlet) also showed an influence of the environment.

\section{AtTempts to PROdUCE NeW GENOTYPICAL QUANTITATIVE} CHARACTERS THROUGH LONG ACTION OF A CERTAIN ENVIRONMENTAL CONDITION

The attempts of the author are largely successful, though in most cases only in their beginnings. He has accomplished a heritable alteration of head-height in the Lunz Untersee Daphnia through sustained overassimilation. At this point he succeeded in "noticeably shifting the reaction norm in the direction of genotypical fixation" of the head height which originally appeared above-normal. This shift yielded three noticeable periods:

In the first period the individuals showed a large variability of the head-form, which was lost in the next molt, when the animals were replaced in the original environment. Also, the head-height returned once more to the old measurement.

In the second period, 3-4 months after the culture's beginning, a singular head-form developed; deformities, aberrations, and doubled structures appeared more seldom. At the same time however, the animals returned more slowly to the original type, when replaced in the original environment. (Young 우우 regressed after more molts, but older animals did not regress.) The offspring born in the original environment show only the original type.

The third period begins almost two years after the initiation of the culture. It is distinguished by the young animals, which, even when they spend their entire development in the original environment, do not return to the original type. They have a higher helm.

A shift of the reaction norm is therefore unmistakeable.

Also, regarding the sexuality an artificial change of the reaction norm in many cases was evident. More races tending towards asexual reproduction completely lost the ability to develop dauer eggs; also for one polycyclic D. pulex the tendency arose to proceed with parthenogenesis even with reduced assimiliation.

I end with a proposition from Johannsen's Elementen der Erblichkeitslehre [sic $]^{13}$ (p. 323): "Briefly stated, the reports from Weismann (and the many other authors more or less under his influence) of fluctuations ${ }^{14}$ as swings in the continuous germplasm variation is wholly unfounded. Overall, when a really exact analysis can be carried through, a genotypical invariance is evident, which is not comportable with the Weismannian speculations regarding determinants."

When one sees how few really exact experiments and observations our judgment is based in as of now for these most important questions of theoretical biology, then one will properly appreciate the worth of Woltereck's culture ex-

\footnotetext{
${ }^{13} \mathrm{MOP}:$ "Elementen der exakten Erblichkeitslehre"

${ }^{14}$ MOP: de Vries and his mutationist followers, including Johannsen, distinguished genotypic differences ("mutations") from environmental/non-genotypic "fluctuating variation".
} 
periments and their conclusions. The continuation of the work announced by the author and their later release in book form will open up a new field of work in freshwater biology of great significance.

Dr. L. Keilhack (Wilmersdorf-Berlin) 